\title{
O circuito arterial da base do encéfalo em suínos (Sus scrofa domesticus Linnaeus, 1758), formação e comportamento
}

Carlos Gomes FERREIRA ${ }^{1}$

Irvênia Luiza de Santis PRADA²

Correspondência para: CARLOS GOMES FERREIRA

Faculdade de Medicina Veterinária

Universidade Federal de Uberlândia

Av. Pará $n^{\circ} 1720$, Bloco $2 T$

Campus Umuarama

38400 - 902 - Uberlândia - MG

Recebido para publicação: 13/11/2002 Aprovado para publicação: 15/03/2005

\author{
1 - Faculdade de Medicina Veterinária da Universidade Federal de \\ Uberlândia, Uberlândia - MG \\ 2 - Departamento de Cirurgia da Faculdade de Medicina Veterinária e \\ Zootecnia da USP, São Paulo - SP
}

\section{Resumo}

Palavras-chave:

Cérebro.

Estudamos em 40 suínos neonatos sem raça definida (SRD), 20

Suínos.

machos e 20 fêmeas, a formação vascular que se dispõe contornando o corpo mamilar, o túber cinéreo, a hipófise e o quiasma óptico na Artérias do encéfalo. Círculo de Willis.

Terezinha município de Uberlândia; 30 deles tiveram seus contingentes arteriais injetados com solução de Neoprene Látex “450”, corada com pigmento específico, tendo sido fixados com solução de formol a 15\% e dissecados; no restante injetou-se Acetato de Vinil corado seguindo-se corrosão em ácido sulfúrico a 30\% os quais eram então lavados em água corrente.Os resultados mostraram que o circuito arterial é uma formação a partir da divisão da artéria carótida do encéfalo em ambos os antímeros, em seus ramos terminais rostral e caudal. Apresenta-se rostralmente em pequeno arco de concavidade medial (antímeros esquerdo e direito); caudalmente apresenta características morfológicas que fazem lembrar figura semelhante a um polígono - metade caudal de um hexágono - (53,3\%) e no restante, figura simbólica de um coração $(46,7 \%)$.O circuito encontra-se fechado rostralmente pela presença constante da artéria comunicante rostral em todas as preparações e, caudalmente, pela presença dos ramos terminais esquerdo e direito da artéria basilar. Em 10\%, além da presença dos ramos terminais esquerdo e direito da artéria basilar, o circuito arterial mostrou no local usual de bifurcação da artéria basilar, duas pequenas anastomoses.

\section{Introdução}

A formação especial estabelecida pelas artérias da base do encéfalo data de 1658 para humanos, por JOHAN WEPTER, cirurgião alemão em seu livro "Historiae Apopleticorum”. Porém, a primeira descrição detalhada dessa estrutura é atribuída ao anatomista inglês Thomas Willis, no ano de 1664, motivo pelo qual ela é classicamente conhecida com "Círculo ou Polígono de Willis".

Desde então, como refere Gonzalez Alvarez $^{2}$, muitos investigadores ficaram impressionados com a extraordinária variedade anatomica desses vasos, tendo sido muitos os trabalhos empreendidos com o objetivo de melhor conhecer o seu comportamento.

Nos animais, trabalhos clássicos como os de Tandler ${ }^{3}$ e De Vriese ${ }^{4}$, assim como Testut $^{5}$, trazem importantes considerações entre a filogenia e a ontogenia dos modelos das artérias encefálicas, assim como sugerem que o sistema nervoso e o correspondente arranjo de seus vasos sangüíneos nas diferentes espécies, encontra-se em dinâmico processo evolutivo. Segundo classificação de Tandler ${ }^{3}$, o círculo de Willis dos mamíferos pode ser agrupado em três tipos 
fundamentais: 1,2 e 3.

Para De Vriese 4 , face à ocorrência de aspectos aparentemente estranhos, inesperados, nas disposicões dessa formação vascular entre os diferentes grupos de mamíferos, o assunto ainda deve ser mais pesquisado. Para Gillilan ${ }^{6}$ os cérebros dos sub-mamíferos vertebrados são supridos por um par de artérias carótidas internas. Gillilan ${ }^{7}$ afirmou que nos mamíferos inferiores um par de artérias curtas fornecem suficiente sangue arterial para suprir ambas partes anteriores e caudais do cérebro, assim o tronco cerebral e o cerebelo recebem maior volume de sangue proveniente do sistema vértebro-basilar deixando o sangue da artérias carótida interna para ser distribuído no cérebro anterior; ainda Gillilan ${ }^{8}$ referiu à ordem Perissodáctila incluindo os cavalos observando que as artérias carótidas internas se dividem em ramos, um rostral maior e um caudal menor e a artéria basilar formada pelas artérias vertebrais esquerda e direita suprindo assim o cérebro.

Mais recentemente tem sido evidenciado grandes variações relacionadas à vascularização arterial do encéfalo entre diferentes espécies animais, como informam de modo geral os tratados de Anatomia Veterinária e de modo particular, trabalhos relativos ao assunto como: Santos ${ }^{9}$ em equinos puro sangue inglês, Santos ${ }^{10} \mathrm{em}$ ovinos, Lindermann ${ }^{11}$ em gambá, Reckziege ${ }^{12} \mathrm{em}$ capivara, Melo ${ }^{13}$ em bovinos azebuados, Alcântara ${ }^{14} \mathrm{em}$ cães sem raça definida, Ferreira ${ }^{15}$ em macaco prego, Ocal et al. ${ }^{16}$ em camelo, Reckziegel, Lindermann e Campos ${ }^{17}$ em capivara, Ferreira e Prada ${ }^{18}$ em macaco prego, Araújo e Campos ${ }^{19} \mathrm{em}$ roedores, Oliveira e Campos ${ }^{20}$ em suínos e Silva e Ferreira ${ }^{21}$ em macaco prego.

Nosso objetivo é o de conhecer nos suínos, uma vez que estes tem sofrido intenso melhoramento genético, particularidades da disposição dessas artérias, podendo assim melhor interpretar aspectos encontrados face à colocações das publicações clássicas já referidas. Os dados obtidos certamente servirão de base para análises comparativas mais amplas entre as várias raças hoje existentes desses animais, assim como entre outras espécies.

\section{Materiais e Métodos}

Utilizamos 40 suínos SRD (sem raça definida), natimortos, provenientes da Fazenda Santa Terezinha, localizada à margem direita na rodovia Uberlândia-Prata, $\mathrm{km} 8$ no município de Uberlândia, Minas Gerais. Em 30 suínos, sendo 15 fêmeas e 15 machos, canulamos a artéria carótida comum esquerda ou direita, e com o auxílio de seringa de 10 $\mathrm{ml}$ nela injetamos solução de Neoprene Làtex "450" corada com pigmento específico. A fixação foi realizada mediante injeção de formol a $15 \%$ pela veia jugular externa de um dos antímeros. Após $24 \mathrm{~h}$ a cabeça com o segmento longo do pescoço foi isolada do resto do corpo. Uma abertura na parede dorsal do crânio foi realizada, visando melhor fixação, sendo então a peça colocada em solução de formol a $15 \%$ por um período mínimo de três dias. Uma vez isolado o encéfalo, procedemos a dissecação com auxílio de Lupa Monocular de luz fria. Utilizamos ainda 10 peças, sendo 5 de fêmeas e 5 de machos, nas quais pela mesma via usada anteriormente, o sistema de vasos da cabeça era "lavado" inicialmente com água a aproximadamente $37^{\circ} \mathrm{C}$. Logo após, injetávamos ar, em seguida Acetona P.A. e, por alguns minutos ar novamente e por fim, solução de Acetato de Vinil corado com corante específico. O material era então colocado em água por $24 \mathrm{~h}$ e em seguida mergulhado em solução de ácido sulfúrico a $30 \%$ por cerca de duas semanas. Daí este foi lavado em água corrente até a limpeza total dos modelos. A nomenclatura por nós utilizada seguiu a International Committee on Veterinary Gross Anatomical Nomenclature. ${ }^{22}$

\section{Resultados}

O circuito arterial do encéfalo representa formação vascular que se dispõe na base do encéfalo, contornando o corpo 
mamilar, o túber cinéreo, a hipófise e o quiasma óptico.

Essa formação ocorre a partir da divisão da artéria carótida do encéfalo em ambos os antímeros, em seus ramos terminais rostral e caudal e dos ramos terminais da artéria basilar; apresenta-se rostralmente em pequeno arco de concavidade medial (antímeros esquerdo e direito); caudalmente apresenta características morfológicas que fazem lembrar figura semelhante a um polígono metade caudal de um hexágono - 53,3\% (Figuras 1 e 3) e no restante, figura simbólica de um coração - 46,7\% (Figuras 2 e 4). Nos casos onde apresentam figura semelhante a um polígono, destacamos a ocorrência de ângulos evidentes em correspondência à
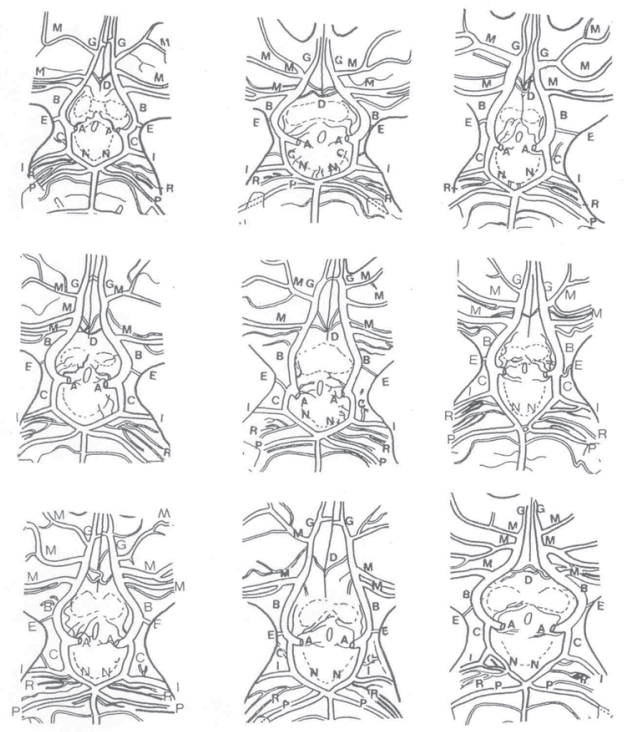

Figura 1 - Esquema das dissecações em que o CIRCUITO ARTERIAL apresenta-se rostralmente em forma de pequeno arco de concavidade medial (antímeros esquerdo e direito) em 100\% dos casos e caudalmente figura semelhante a um polígono - metade caudal de um hexágono (53,3\%). A artéria carótida do encéfalo (A) de cada antímero divide-se em um ramo rostral (B) e outro caudal (C). Do primeiro origina-se em cada antímero as artérias cerebral rostral (G), média (M), corioidea rostral (E) e comunicante rostral (D). Do segundo, origina-se em cada antímero a artéria cerebral caudal (I). Do ramo terminal da artéria basilar (N) em cada antímero origina-se as artérias mesencefálicas $(R)$ e algumas vezes também a artéria cerebelar rostral $(\mathrm{P})$ origem da artéria cerebral caudal (Figura 1) e ramos terminais da artéria basilar (Figura 3, J) em ambos antímeros.

O circuito encontra-se fechado rostralmente pela presença constante da artéria comunicante rostral em 100\% dos casos e caudalmente, pela presença dos ramos terminais esquerdo e direito da artéria basilar (Figuras 1, 2, 3 e 4). Em algumas situações $(10 \%)$, além da presença dos ramos terminais esquerdo e direito da artéria basilar, o circuito arterial encontra-se fechado também por duas pequenas anastomoses que se encontram no local usual de bifurcação de seus ramos terminais. Desses, em um caso $(3,3 \%)$ no antímero direito, a artéria basilar continua-se no vaso representativo de seu ramo terminal direito. No antímero esquerdo o vaso
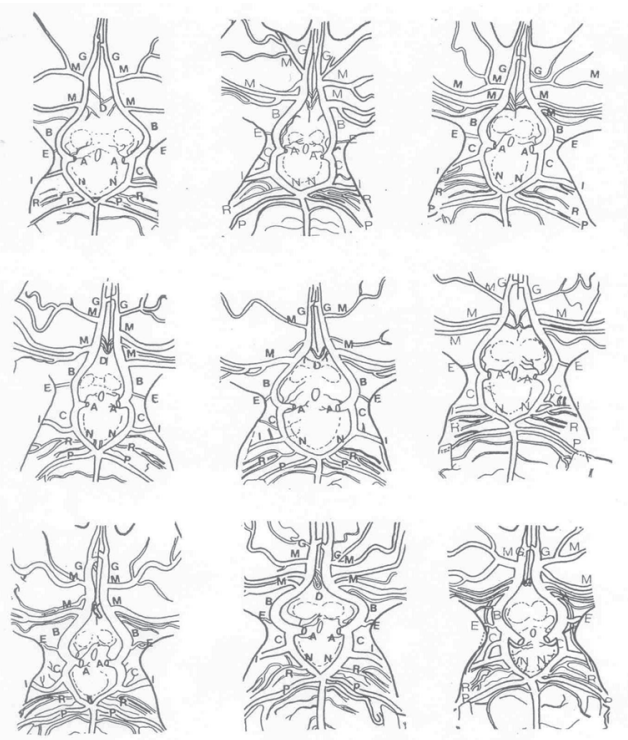

Figura 2 - Esquema das dissecações em que o CIRCUITO ARTERIAL apresenta-se rostralmente em forma de arco de concavidade medial (antímeros esquerdo e direito) em $100 \%$ dos casos e caudalmente figura simbólica de um coração $(46,7 \%)$. A artéria carótida do encéfalo de cada antímero (A), divide-se em ramos rostral (B) e caudal (C). Do primeiro origina-se em cada antímero as artérias, cerebral rostral $(\mathrm{G})$, média $(\mathrm{M})$, corioidea rostral (E) e comunicante rostral (D). Do segundo, origina-se em cada antímero a artéria cerebral caudal (I). Do ramo terminal da artéria basilar $(\mathrm{N})$, em cada antímero, origina-se as artérias mesencefálicas (R) e algumas vezes também a artéria cerebelar rostral $(\mathrm{P})$ 
representativo de seu ramo terminal, parece continuar-se diretamente da artéria cerebelar rostral; nesta situação, entre os dois antímeros, na origem da artéria cerebelar rostral, ocorrem duas anastomoses de disposição transversal. Nos outros dois casos ocorre o inverso; no antímero esquerdo, a artéria basilar parece continuar-se diretamente no seu ramo terminal. No antímero direito, o vaso representativo do seu ramo terminal sugere continuar-se diretamente na artéria cerebelar rostral, observando a presença das duas referidas anastomoses transversais, estando nestes casos a mais rostral, implicada no fechamento da porção caudal do circuito arterial do encéfalo.

\section{Discussão}

O circuito arterial, que observamos na base do encéfalo dos suínos estudados, resultou da particular disposição dos ramos

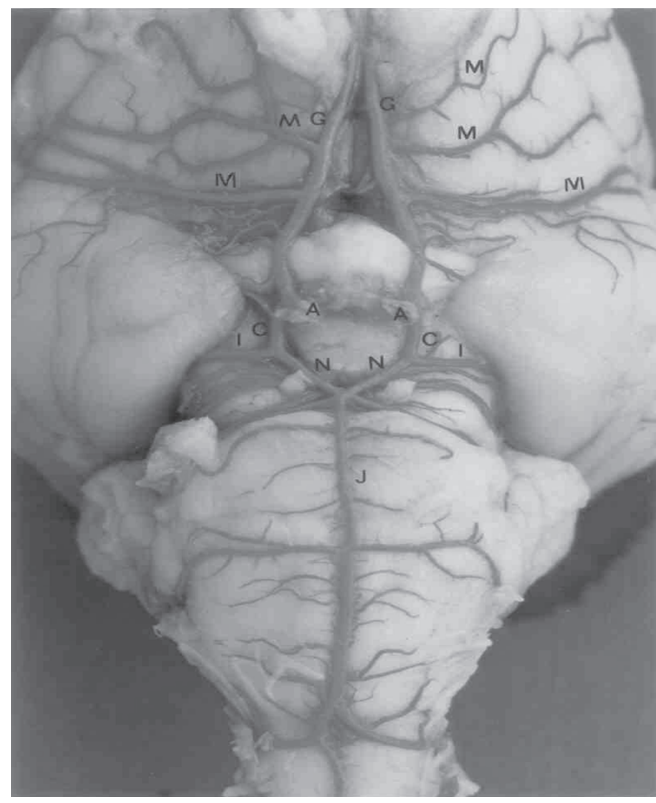

Figura 3 - Encéfalo de suíno sem raça definida. Aumento de aproximadamente 2,2 vezes. O CIRCUITO ARTERIAL acha-se representado caudalmente por figura semelhante a um polígono - metade caudal de um hexágono $(53,3 \%)$. Identificamos ainda a artéria basilar $(\mathrm{J})$, seus ramos terminais $(\mathrm{N})$ unindo-se ao ramo caudal (C) da artéria carótida do encéfalo (A) no limite referendado pela origem da artéria cerebral caudal (I). Artéria cerebral média (M), artéria cerebral rostral $(G)$

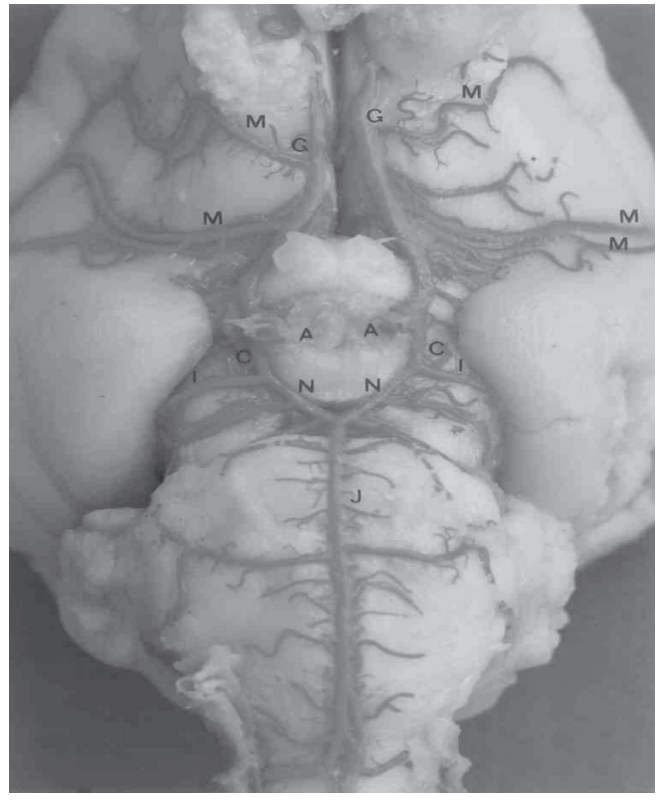

Figura 4 - Encéfalo de suíno sem raça definida. Aumento de aproximadamente 2,2 vezes. O CIRCUITO ARTERIAL acha-se representado caudalmente por figura simbólica de um coração $(46,7 \%)$. Identificamos ainda a artéria basilar $(\mathrm{J})$, seus ramos terminais $(\mathrm{N})$ unindo-se ao ramo caudal (C) da artéria carótida do encéfalo (A) no limite referendado pela origem da artéria cerebral caudal (I). Artéria cerebral média $(\mathrm{M})$, artéria cerebral rostral (G)

rostral e caudal da artérias carótidas internas e dos ramos terminais da artéria basilar. A figura que encontramos na maioria dos casos, como referiu Alcântara ${ }^{14} \mathrm{em}$ cães, não corresponde em seu todo nem a um polígono arterial como Baptista ${ }^{23}$ refere, nem a um hexágono como citam Ellenberger e Baum $^{24}$, nem tampouco a um círculo arterial ou arterioso a que aludem De Vriese ${ }^{4}$ e Nickel, Schummer e Seiferle ${ }^{25}$, entre outros.

A formação que descrevemos, observado também por $\mathrm{Nanda}^{26}$, apresentase em geral, rostralmente em pequeno arco de concavidade medial (antímeros esquerdo e direito). Caudalmente mostra características morfológicas que lembram um polígono (metade caudal de um hexágono) ou a figura simbólica do coração. Essa situação não foi referida por nenhuma literatura consultada. Preferimos assim como Alcântara ${ }^{14}$, adotar a expressão circuito arterial uma vez que a mesma, conforme definição feita por Ferreira $^{27}$, atende à figura por nós encontrada. 
Baptista ${ }^{23}$ refere-se à estrutura como círculo. Segundo o autor, este círculo dispõe-se em torno da sela túrcica e se encontra na superfície ventral do encéfalo, informando que este círculo está constituído caudalmente e rostralmente, pelas mesmas estruturas por nós encontradas, apesar de utilizarem nomenclatura diferente para os vasos implicados. Com relação à denominação dos vasos da base do encéfalo a tendência da literatura foi por um longo período considerar o modelo humano como padrão para muitas classes animais, mas particularmente para os suínos esta arquitetura difere em vários aspectos.

Em relação à parte caudal do circuito, Bruni e Zimmerl ${ }^{28}$, fazem referência à artéria comunicante posterior circundando o corpo mamilar, anastomosando-se com sua homóloga do antímero oposto. Também Nanda $^{26}$ cita a artéria comunicante caudal situada entre a artéria carótida e a artéria basilar. Entretanto, de acordo com nossos resultados, isto não se aplica aos suínos em que os dois sistemas constituem uma única unidade vascular, centralizada no circuito arterial. Ao contrário do apontado por Ferreira $^{15}$ e Shellshear ${ }^{29,30}$ em referência aos macacos do novo e do velho mundo, esta pesquisa em suínos sinaliza existir continuidade de fluxo entre os sistemas carótico e occipito-basilar. É interessante, o fato de, por vezes, o calibre do segmento mais rostral da artéria basilar ser inferior ao de seus ramos terminais diferentemente do apontado por Ferreira e $\operatorname{Prada}^{18}$ e Siqueira Neto e Ferreira ${ }^{31}$ em macaco prego no qual a artéria basilar tem o calibre equivalente ao dobro de seus vasos formadores e de seus ramos terminais. O circuito arterial do encéfalo dos suínos pode ser interpretado como um complexo anastomótico de vascularização à semelhança do aventado por $\mathrm{Kramer}^{32}$ para cães e macacos, por Shellshear ${ }^{29}$ e Ferreira ${ }^{15}$ para macacos e por Rogers $^{33}$ para o homem, ressaltando-se o potencial das anastomoses em artérias que vascularizam estruturas exigentes quanto ao aporte sangüíneo, como é o caso do encéfalo.
$\mathrm{Na}$ capivara por sua vez Recziegel, Lindermann e Campos ${ }^{17}$ documentaram um modelo arterial com predominância expressiva em termos de hierarquia de calibre dos vasos do circuito anastomótico caudal. Do ponto de vista ontogenético e filogenético, podemos dizer que estas anastomoses são estáveis, uma vez presentes em todas as observações em ocorrência de anomalias, hipoplasias ou hiperplasias e também ausência de vasos.

As artérias da base do encéfalo, ao constituírem o circuito arterial do encéfalo dispõem-se de maneira a caracterizarem situação intermediária entre os estágios médio e final referidos por Testut. ${ }^{5}$ Entretanto, ao tentar inserir o padrão de comportamento das artérias encefálicas dos suínos, na classificação de Tandler ${ }^{3}$, referida por De Vriese $^{4}$, diferentes aspectos desse comportamento mostram-se quase sempre discordantes entre si na caracterização de um tipo específico. Assim, o padrão suíno estaria concordante com o tipo 1 apenas em relação ao comportamento do ramo rostral da artéria carótida interna, não correspondendo a esse tipo quanto à disposição do ramo caudal, pois no caso, este não constitui a artéria basilar e nem esta mostra calibre decrescente rostrocaudal. Também não corresponde totalmente ao tipo 2 alfa, em que as três artérias cerebrais (rostral, média e caudal), originam-se da artéria carótida interna (artéria carótida do encéfalo, para nós), pois não temos como afirmar que em nosso material a artéria cerebral caudal realmente resulta do ramo caudal da artéria carótida do encéfalo. Dado a isto, consideramos a origem da artéria cerebral caudal no ponto de encontro do ramo caudal da artéria do encéfalo com o correspondente ramo terminal da artéria basilar. Também caracterizando o tipo 2 alfa, De Vriese 4 , considera os "ramos de divisão da artéria basilar"o que igualmente levamos em conta principalmente dado o fato de que essa artéria mostrou diminuição progressiva de calibre caudo-rostral. Também nem sempre o calibre desses ramos é inferior ao calibre da artéria basilar, o que, a rigor seria 
esperado. Por outro lado, o padrão suíno também não se coaduna com o tipo 2 beta. Assim sendo, julgamos que o padrão suíno das artérias estudadas não se insere nos tipos 1 e 2 beta e também não se enquadra totalmente no tipo 2 alfa. Poderíamos dizer que esse padrão tende ao tipo 2 alfa. Vale aqui considerar que os resultados dessa pesquisa não equivalem ao apontado por De Vriese $^{4}$ como modelo de vascularização para artiodáctilos e perissodáctilos. A autora referiu-se possivelmente de maneira genérica a esses grupos animais, sem sustentar suas conclusões em trabalhos com amostragem mais substantiva. Ficaram portanto como pertencendo ao tipo 1 referido por Tandler. ${ }^{3}$ Os nossos resultados se aproximam muito mais dos modelos característicos de alguns carnívoros, como refere De Vriese. ${ }^{4}$ Assim, grupos aparentemente distantes podem, como mesmo referiu a autora, na sua opção ou pressão evolutiva, ter permanecido em investimentos semelhantes na forma, o que não deixa de caracterizar a riqueza do repertório biológico. Por outro lado, podemos considerar que em termos de adaptação evolutiva pelo processo de domesticação, o homem e o suíno tem uma longa história de vida em comum e sofreram pressões de seleção em nichos e habitats comuns, disputando inclusive hábitos alimentares parecidos; estas populações cruzaram um vale adaptativo muito semelhante, o que para Futuyma (1993 apud FERREIRA, 1997, p.
16-17), pode significar a transposição de um pico adaptativo para outro.

\section{Conclusões}

A particular disposição dos ramos das artérias carótidas do encéfalo esquerda e direita e dos ramos terminais da artéria basilar, determina a formação do circuito arterial do encéfalo, que contorna o quiasma óptico e a hipófise. Essa formação que, no seu conjunto, não corresponde nem a um círculo, nem a um polígono, apresenta-se de modo invariável, rostralmente, em pequeno arco de concavidade medial (antímeros esquerdo e direito) e, caudalmente em forma de polígono - metade caudal de um hexágono $(53,3 \%)$ ou em figura simbólica do coração $(46,7 \%)$.

É observada a ocorrência de anastomoses entre os ramos terminais da artéria basilar $(10,0 \%)$, mais precisamente no ponto de transição entre o tronco da mesma e os seus respectivos ramos, contribuindo com isso para o fechamento caudal do circuito arterial nesses casos. Rostralmente o circuito encontra-se fechado pela presença constante da artéria comunicante rostral.

O padrão vascular das artérias da base do encéfalo dos suínos estudados tende ao tipo 2 alfa referido por De Vriese $^{6}$ e situa-se entre os estágios médio e final de seu desenvolvimento filogenético, considerados por Testut ${ }^{34}$.

\section{The brain arterial circuit in swine (Sus scrofa domesticus Linnaeus, 1758), behaviour and formation}

\section{Abstract}

Forty neonatal pigs were usec both (male and female) in the amount of 20 each in order to study the vascular arrangement that is placed in the region of the mammillary corpus, the tuber cinereous, the hypophysis and the optical chiasma in the encephalon basis. All the animals were from Santa Terezinha Farm sited in Uberlândia. 30 of them had their arteries injected with Neoprene Latex "400" colored with a specific pigment and then fixed with 15\% formaldehyde solution. After that, they were dissected. The $10 \mathrm{left}$ were injected with colored vinyl acetate followed by corrosion with $30 \%$ sulfuric acid solution. The pieces were washed afterward in
Key-words:

Brain.

Pigs.

Encephalon arteries. Willis circuit. 
tap water. The results showed that the arterial circuit is a formation from the division of encephalon carotid artery in both hemisphere in its rostral and caudal terminal branches. The appearance of the arterial circuit is rostrally like a small medial concavity arc (left right halves). Caudally it shows morphologic characteristics as a polygon picture - a hexagonal caudal half - $(53.3 \%)$ and the left, like a heart picture $(46.7 \%)$. The circuit is rostrally closed by the constant presence of the rostral communicant artery in all the preparations and caudally by the presence of left and right terminal branches of basilar artery. Besides the presence of left and right terminal branches of basilar artery, in $10 \%$ of the pieces the arterial circuit showed at the usual place of bifurcation, two little anastomosis.

\section{Referências}

1 BARBOSA, L. L.; PURRIEL, J. A.; MEERHOFF, W.; MEDOC, I. El polígono de Willis y sus variaciones. Acta Neurologica Latinoamericana, Montevideo, v. 15, p. 224-236, 1969.

2 GONZALEZ ALVAREZ, A. Circulo arterial de Willis Sus medidas externas y variaciones anatómicas. Acta Neurologica Latinoamericana, Mendoza, v. 5, p. 1-6, 1959.

3 TANDLER, J. Zur Vergleichenden Anatomie der Kopfarterien bein den Mammalia. Denkschr. Akad. Wiss. Wien, v. 67, p. 677-784, 1898.

4 DE VRIESE, B. Sur la significtion morfologique des artères cerébrales. Archives de Biologie, Liège, v. 21, p. 357-457, 1905.

5 TESTUT, L. Traité $\mathbf{d}$ anatomie humaine. 6. ed. Paris: Octave Doin, 1911. v. 2, p. 916-920.

6 GILLILAN, L. A. A Comparative study of the extrinsic and intrinsic arterial blood supply to brains of submammalian vertebrates. J. Comp. Neur., v. 130, p. 175-196, 1967.

7 GILLILAN, L. A. Blood supply to primitive mammalian brains. J. Comp. Neur., v. 145, p. 209-222, 1972.

8 GILLILAN, L. A. Blood supply to brains of ungulates with and without a rete mirabile caroticum. J. Comp. Neur., v. 153, p. 275-290, 1974.

9 SANTOS, A. Estudo anatômico das artérias da base do encéfalo de eqüinos da raça puro sangue inglês. 1987. 111 f. Dissertação (Mestrado em Anatomia) - Faculdade de Medicina Veterinária e Zootecnia, Universidade de São Paulo, São Paulo, 1987.

10 SANTOS, R. M. B. Estudo anatômico das artérias da base do encéfalo em ovinos (Ovis aries, L., 1758). 1993.
110 f. Tese (Doutorado em Anatomia) - Faculdade de Medicina Veterinária e Zootecnia, Universidade de São Paulo, São Paulo, 1993.

11 LINDERMANN, T. Sistematização da irrigação na base do encéfalo do gambá (Didelphis albiventris). 1994. 127 f. Dissertação (Mestrado em Anatomia) - Faculdade de Veterinária, Universidade Federal do Rio Grande do Sul, Porto Alegre, 1994.

12 RECKZIEGEL, S. H. Sistematização da irrigação na base do encéfalo de capivara (Hydrochoerus hydrochoeris). 1994. 133 f. Dissertação (Mestrado em Anatomia) - Faculdade de Veterinária, Universidade Federal do Rio Grande do Sul, Porto Alegre, 1994.

13 MELO, A. P. F. Estudo anatômico das artérias da base do encéfalo em fetos de bovinos azebuados. 1996. 106 f. Dissertação (Mestrado em Anatomia) - Faculdade de Medicina Veterinária e Zootecnia, Universidade de São Paulo, São Paulo, 1996.

14 ALCÂNTARA, M. A. Artérias da base do encéfalo de cães (Canis familiaris LINNAEUS, 1758). II. Formação e comportamento do circuito arterial do encéfalo. Braz. J. vet. Res. Anim. Sci., v. 33, n. 2, p. 72-76, 1996.

15 FERREIRA, J. R. Estudo anatômico das artérias da base do encéfalo do macaco prego (Cebus apella, LINNAEUS, 1766). 1997. 192 f. Tese (Doutorado em Anatomia) - Faculdade de Medicina Veterinária e Zootecnia, Universidade de São Paulo, São Paulo, 1997.

16 OCAL, M. K. et al. Quantitative study of the circulus arteriosus cerebri of the Camel (Camelus dromedarius). Anat. Histol. Embryol., v. 28, p. 271-272, 1999.

17 RECKZIEGEL, S. H.; LINDERMANN, T.; CAMPOS, R. A systematic study of the brain base arteries in capybara (Hydrochoerus hydrochoeris). Braz. J. Vet. Res. Anim. 
Sci., v. 18, n. 2, p. 103-110, 2001.

18 FERREIRA, J. R.; PRADA, I. L. S. Nomenclatura proposta para denominar as artérias da base do encéfalo do macaco prego (Cebus apella L., 1766). Acta Scientiarum, v. 23, n. 2, p. 635-643, 2001.

19 ARAÚJO, A. C. P.; CAMPOS, R. A systematic study of the brain base arteries in chinchila. Braz. J. of vet. Res. Anim. Sci., v. 18, n. 2, p. 139-140, 2001.

20 OLIVEIRA, J. C. D.; CAMPOS, R. The rostral and caudal epidural rete mirabile and its supply sources in wild board (Sus scrofa). Braz. J. Vet. Res. Anim. Sci., v. 18, n. 2, p. 185, 2001.

21 SILVA, R. A.; FERREIRA, J. R. Morfologia da artéria cerebelar superior do macaco prego (Cebus apella L., 1766): divisões e anastomoses. Acta Scientiarum, v. 24, n. 3, p. 687-695, 2002.

22 INTERNATIONAL COMMITTEE ON VETERINARY GROSS ANATOMICAL NOMENCLATURE. Nomina anatomica veterinaria. 4. ed. Zurich, 1994. (Together with nomina histological, 2. ed., 1992 and nomina embriologica veterinary, 1992).

23 BAPTISTA, B. V. Estudo comparado da circulação cerebral dos mamíferos domésticos e no homem - Razão de ser da rede admirável. 1922. 89 f. Tese (Doutorado em Anatomia) - Faculdade de Medicina do Rio de Janeiro, Rio de Janeiro, 1992

24 ELLENBERGER, W.; BAUM, H. Handbuch der Vergleichenden Anatomie der Haustiere. Berlim: Springer-Verlag, 1977. p. 669-671.

25 NICKEL, R.; SCHUMMER, A.; SEIFERLE, E. Nervensystem Sinnesorgane endokrine Drusen. Berlim: Paul Parey, 1975. v. 4. p. 174-181.

26 NANDA, B. S. Suprimento sangüíneo para o cérebro. In: GETTY, R. Sisson/Grossman anatomia dos animais domésticos. 6. ed. Rio de Janeiro: Guanabara Koogan, 1986. v. 2, p. 1232-1237.

27 FERREIRA, A. B. H. Novo dicionário da língua portuguesa. 2. ed. Rio de Janeiro: Nova Fronteira, 1986. p. 410.

28 BRUNI, A. C.; ZIMMERL, U. Anatomia degli animali domestici, Milano: Francesco Vallardi, 1947. v. 2, p. 319-324.

29 SHELLSHEAR, J. L. The arteries of the brain of the Orang-Utan. J. Anat., v. 61, p. 167-197, 1927.

30 SHELLSHEAR, J. L. The arterial supply of the cerebral cortex in the Chimpanzee (Anthropithecus troglodytes).
J. Anat., v. 65, p. 45-87, 1930.

31 SIQUEIRA NETO, E. G. B.; FERREIRA, J. R. Estudo anatômico da origem e distribuição dos ramos corticais das artérias cerebrais caudais do encéfalo do macaco prego (Cebus apella Linnaeus, 1766). Acta Scientiarum, v. 24, n. 2, p. 639-646, 2002.

32 KRAMER, S. P. On the function of the circle of Willis. Journal of Experimental Medicine, v. 15, p. 348-355, 1912.

33 ROGERS, L. The function of the circulus arteriosus of Willis. Brain, v. 70, p. 171-178, 1947. 